\title{
Bioleta: collaborative mapping and a fairer trade for artisans
}

\author{
Gal Villaseñor Ortega, Georgina Cantú Muñoz, Valeria Pérez Meraz, Rocío \\ Abascal Mena
}

Published: 30 November 2020

\begin{abstract}
BIOLETA is an interdisciplinary proposal, developed by using the User-Centered Design (UCD) approach, that aims to create a collaborative network to promote fair trade and the revalorization of artisanal work through an app that facilitates the sale of artisan's products without intermediaries, which is a win-win for artisans and clients. The app, named BIOLETA for the biodiversity that artisanal work requires, has two goals: to create a reliable database of Mexican crafts after the self-registration of artisans from all over the country and therefore to facilitate the direct contact and relationship of artisans with buyers and institutions. The second goal is to generate a collaborative mapping of street vendors of artisanal work that do not have a digital presence. With the help of potential buyers, neighbors, and/or people interested in artisanal work, the "mapper" will introduce the possible geolocations, recommendations and if available, the contact information of the street vendors to increase their sales during -and after- the emergency caused by COVID- 19 .
\end{abstract}

\section{Keywords}

Collaborative network, artisanal work, User-Centered Design, prototype, contextual observation.

\section{Introducción}

La pandemia del COVID-19 trajo una crisis mundial sin precedentes, la cual está exponiendo y agravando las desigualdades de las sociedades. Este artículo se centra en cómo la emergencia afecta a los vendedores de trabajo artesanal, que están en las calles, y en cómo crear una solución que permita aumentar las ventas y la apreciación de su trabajo.

La Organización Internacional del Trabajo (OIT) [11] advierte que los 1,6 mil millones de trabajadores en la economía informal casi la mitad de la fuerza mundial- corren un peligro inmediato de que sus medios de subsistencia se vean seriamente afectados después de los acontecimientos de 2020. En México, el panorama

\footnotetext{
Villaseñor Ortega, G., Cantú Muñoz, G., Pérez Meraz, V., Abascal

Mena, R.

Universidad Autónoma Metropolitana - Cuajimalpa

Ciudad de México, México.

galvillasenor@gmail.com, georginacantu91@gmail.com,

vlaleria@gmail.com,mabascal@cua.uam.mx
}

es especialmente preocupante, de acuerdo con el Instituto Nacional de Estadística y Geografía (INEGI) el $56.2 \%$ de los trabajadores mexicanos son parte del sector informal, donde no hay derechos fundamentales garantizados [8].

Los vendedores ambulantes de artesanías son uno de los grupos más afectados porque la mayoría vive de sus ventas diarias. Incluso, antes de la situación actual causada por COVID-19, los vendedores ambulantes de trabajo artesanal han sido uno de los grupos más vulnerados. Muchos de ellos, son parte de las comunidades indígenas de otros estados del país que migran a la Ciudad de México en busca de crecimiento económico y oportunidades de empleo. Asimismo, se enfrentan a la discriminación y al menosprecio de su trabajo artesanal, que requiere una gran cantidad de práctica y horas de trabajo, además de ser objetos de transmisión de memoria y parte del patrimonio cultural del país.

El resto del artículo está organizado de la siguiente manera: la sección 2 está destinada a dar un contexto sobre la importancia del trabajo artesanal como una manifestación intangible de la cultura patrimonial y los desafíos a los que los artesanos se enfrentan, incluyendo la brecha digital en la economía digital prominente. En esta sección, también se reconocen algunas plataformas que utilizan las nuevas tecnologías para lograr un comercio más justo para los artesanos y, en algunos casos, muestran la relevancia del trabajo colaborativo a través del mapeo. La sección 3 se centra en la metodología de la creación de BIOLETA: detección de necesidades, definición del problema, propuesta de solución y evaluación. El primer paso, consistió en la observación digital llamada netnografía y la identificación de la máxima necesidad de los artesanos: tener ventas a pesar de la cuarentena. Después, se utilizaron varias herramientas provenients del Diseño Centrado en el Usuario (DCU): storyboards, personas y paneles de inspiración para poder elegir una necesidad y crear un prototipo de solución. Para esta parte del proceso y debido a la cuarentena, se utilizó la técnica del pretotipado antes de realizar el prototipo final. El pretotipo permitió validar la idea antes de la construcción de un prototipo digital. Finalmente, el pretotipo fue evaluado a distancia con las 10 heurísticas propuestas por Jakob Nielsen [14].

La sección 4 muestra los resultados del pretotipo y la posibilidad de hacer de BIOLETA un proyecto integral en el cual la tecnología pueda ser una herramienta que una a las personas y mejore el futuro de la comunidad artesanal mexicana $\mathrm{e}$ históricamente marginada. 


\section{2 ¿Qué es el trabajo artesanal y por qué es tan importante?}

Un artesano es alguien que trabaja con sus manos para crear artículos únicos (funcionales y/o decorativos) utilizando técnicas tradicionales aprendidas, a través de décadas de conocimiento ancestral, y transmitidas dentro de las familias y las comunidades [19]. En términos económicos, un artesano es un pequeño productor de bienes que posee su producción y que se gana la vida con su comercio.

Como se cita en el Manual de diferenciación entre artesanía y manualidad del Fondo Nacional para el Fomento de las Artesanías (FONART) [5], las artesanías no sólo se definen por su valor estético en el dominio de la transformación técnica y la ornamentación, sino que, para ser consideradas como tal, deben representar valores simbólicos e ideológicos de la sociedad que los crea. Contrario a lo anterior, las manualidades se rigen en los tiempos presentes y tienden a la estandarización de su producción con los fenómenos de la globalización y la cultura de masas.

En México, los productos hechos a mano con fines utilitarios y el arte popular se conocen colectivamente como artesanía. Las artesanías mexicanas tienen sus fundamentos en los oficios de las culturas prehispánicas, pero las influencias europeas las transformaron en una mezcla de ambas culturas y se han convertido en una importante parte de la identidad nacional.

Muchos artesanos dependen de los recursos de su entorno para crear estos artículos y son "maestros" de su oficio. Algunos ejemplos de esto son: la cerámica, los textiles, la madera, la metalurgia, la joyería, el uso de fibras naturales, cartón y papel, y el vidrio.

A nivel mundial, México es el tercer país con la mayor producción artesanal, la gama de artesanías es tan diversa que sería imposible contar con la cantidad exacta de técnicas que existen en el país. El Estado de Hidalgo es el mayor productor de artesanía, seguido por Oaxaca y Michoacán. Según el INEGI, 500,754 personas en México se dedican a la industria artesanal, 141,949 $(28.35 \%)$ son mujeres y $358,805(71.65 \%)$ son hombres [18]. Oaxaca es el estado con más artesanos con 58,398 en total, seguido por Guerrero con 39,107 trabajadores, y el Estado de México con 34,687 [7].

Los artesanos enfrentan muchos obstáculos: la globalización y el desafío de la producción en masa, la supervivencia de las formas tradicionales de artesanía y la ausencia de medidas legales tales como protección a la propiedad intelectual, derechos de autor o registros de copyright. Esto los deja en una situación desfavorecida, ya que necesitan competir con el bajo costo y a menudo mala calidad que ofrece la producción en masa. Asimismo, tiendas de moda rápida como Zara y marcas de alta gama como Carolina Herrera, Isabel Marant, y Dior han copiado la iconografía indígena y los textiles, sin ningún reconocimiento o remuneración a las comunidades que originalmente lo crearon [6].

La mayoría de los artesanos venden en lugares informales, como los mercados o las calles, y encima de enfrentar todas las desventajas de un empleo informal. También sufren la falta de comprensión del valor simbólico de su trabajo, además, experimentan una gran cantidad de negociación y regateo. Una encuesta llevada a cabo por la Red de Artesanos y Productores, en

\footnotetext{
${ }^{1}$ https://www.map.cdmx.gob.mx/

2 https:/www.gob.mx/fonart/

${ }^{3} \mathrm{https}: / /$ www.lourdesarizpe.com/
}

la que 285 artesanos del centro de México participaron, mostró que entre el $85 \%$ y el $90 \%$ de las personas que quieren comprar una pieza de artesanía intenta bajar el precio [17]. Según la misma encuesta, el "descuento" que solicitan las personas oscila entre el $25 \%$ y el $30 \%$, y debido a la necesidad urgente de vender, muchas veces los artesanos se ven obligados a aceptar este precio reducido. El regateo sucede en muchos países de todo el mundo, e incluso, algunas personas consideran que es cultural. Sin embargo, es importante dar a conocer la importancia del trabajo artesanal y su valor para eliminar esta práctica.

\subsection{La Brecha Digital: una Realidad en México}

El mundo nunca estuvo tan interconectado como ahora. En 2020, las Tecnologías de la Información y la Comunicación (TICs) han sido una parte vital de la dinámica social, cultural y económica. Incluso, antes de COVID-19, ser excluido del mundo digital significaba quedar fuera de muchas actividades de la vida diaria y no verse beneficiado por el alcance de la tecnología [13]. La pandemia no solo ha acelerado el uso de soluciones digitales y la transición global hacia una economía digital, sino que también ha puesto en relieve la brecha que ya existía entre las personas con posibilidades de acceso a la tecnología de infraestructura y dispositivos, y los que se han quedado al margen, ya sea debido a la falta de acceso a las TICs o falta de conocimientos sobre cómo usarlos $[3,21]$.

De acuerdo con el INEGI, existen en México 71.3 millones de usuarios con conexión a Internet, lo que representa casi el $63.9 \%$ de la población a partir de los seis años [9]. Sin embargo, la brecha es aún notable, especialmente en las zonas rurales. Por ejemplo, en el Estado de Oaxaca, el $60 \%$ de los hogares no tiene acceso a un teléfono móvil [9]. Los datos muestran que incluso cuando existe la infraestructura para utilizar un teléfono celular, (con o sin un plan de datos para el acceso a Internet), un tercio de los hogares no tienen la posibilidad económica para hacerlo, sobre todo en los estados más pobres.

Es necesario trabajar para eliminar las desigualdades digitales desde un enfoque estructural, sin embargo, como se explica en la conclusión, la propuesta de BIOLETA es solo un primer paso para resolver un problema que requiere de una solución urgente como lo estableció Cultural Survivor en la Cumbre Mundial sobre la Sociedad de la Información (WSIS) "la dignidad y los derechos humanos de los pueblos indígenas, naciones y tribus, ..., deben ser afirmados si la brecha en términos de economía, información y digital, separa a las naciones ricas en tecnología y el sector privado de los pueblos más marginados de la sociedad, incluidos los pueblos indígenas, las naciones y las tribus” [12].

\subsection{Trabajo Relacionado}

Existen diversas instituciones como el Museo de Arte Popular $(\mathrm{MAP})^{1}$ y el Fondo Nacional para el Fomento de las Artesanías $(\text { FONART })^{2}$ dedicados a la promoción de las artesanías, además de varios teóricos que han profundizado en su producción académica acerca de la importancia simbólica de las artesanías y el patrimonio cultural mexicano: Lourdes Arizpe ${ }^{3}$, Néstor García Canclini ${ }^{4}$ y Marta Turok ${ }^{5}$, por mencionar a algunos. Sin embargo, existe poca literatura acerca de las nuevas tecnologías y sus posibilidades de colaboración con las artesanías.

\footnotetext{
${ }^{4}$ https://www.ses.unam.mx/ciij/ngc.htm

${ }^{5} \mathrm{https}: / / 17$ edu.org/marta-turok/
} 
Por otro lado, hay algunas aplicaciones que tratan de resolver diferentes problemas y que tienen cierta similitud con el objetivo principal de BIOLETA que es vender más y valorizar la producción artesanal.

- RENATRA es el Registro Nacional de Artesanos Textiles de la República de Argentina. RENATRA registra a los artesanos textiles para reflejar la diversidad que caracteriza la producción del país y generar datos públicos y oficiales. Permite a los artesanos llevar su trabajo a un amplio consumidor público, a la vez que facilita la creación de vínculos y redes entre los propios artesanos [16]. A diferencia de BIOLETA, no incluye a los artesanos que no tienen una presencia digital.

- $\quad$ Muymuymexicano ${ }^{6}$ es un sitio web de comercio electrónico que solo vende productos mexicanos creados por empresarios que venden piezas únicas hechas a mano. Al igual que BIOLETA, la atención se centra en los productos mexicanos, sin embargo, la presente propuesta no pretende ser un comercio electrónico o vender de forma directa.

- $\quad$ OpenStreetMap es un proyecto colaborativo que permite la creación de mapas editables del mundo de forma gratuita. Vinculados a OSM han surgido iniciativas como GeoChicas (@GeochicasOSM), un grupo de mujeres que notaron que existía un problema estructural en las comunidades debido a la gestión de datos y a la falta de participación femenina y liderazgo de proyectos. Es un buen ejemplo de cómo el mapeo colaborativo puede tener un impacto social, aunque no se centran en el trabajo artesanal tradicional.

- $\quad M a p s . m e^{8}$ es una plataforma en la que es posible descargar mapas sin tener acceso a Internet. No tiene muchas similitudes con BIOLETA porque no hace el trabajo con productos artesanales, sin embargo, es un ejemplo de mapeo inclusivo.

- Grupos de Facebook son grupos auto-organizados de gente que quiere comprar o vender productos artesanales, pero no hay ninguna regulación ni una base de datos fiable.

Después de una amplia investigación, no fue posible encontrar proyectos similares a BIOLETA. Las principales diferencias radican en el hecho de que BIOLETA no es una tienda de comercio electrónico, sino una solución de colaboración, que prioriza el tiempo que se dedicó al trabajo sobre el precio final, que se centra únicamente en trabajo artesanal tradicional y elimina intermediarios. BIOLETA lucha contra la brecha digital al proponer soluciones alternativas como el mapeo de los artesanos y sus productos artesanales.

\section{Metodología}

El desarrollo de BIOLETA, como se puede ver en la Figura 1, se realizó mediante el uso del enfoque del DCU, un proceso definido por la Interaction Design Foundation como "un proceso de diseño iterativo en el que los diseñadores y otras partes interesadas se centran en los usuarios y sus necesidades en cada fase del proceso de diseño, permitiendo la participación de los usuarios en todo el proceso de diseño a través de una variedad de técnicas de investigación y de diseño para crear productos usables y accesibles para ellos" [10].

\footnotetext{
${ }^{6} \mathrm{https}: / /$ www.muymuy.mx/

${ }^{7}$ https://www.openstreetmap.org/
}

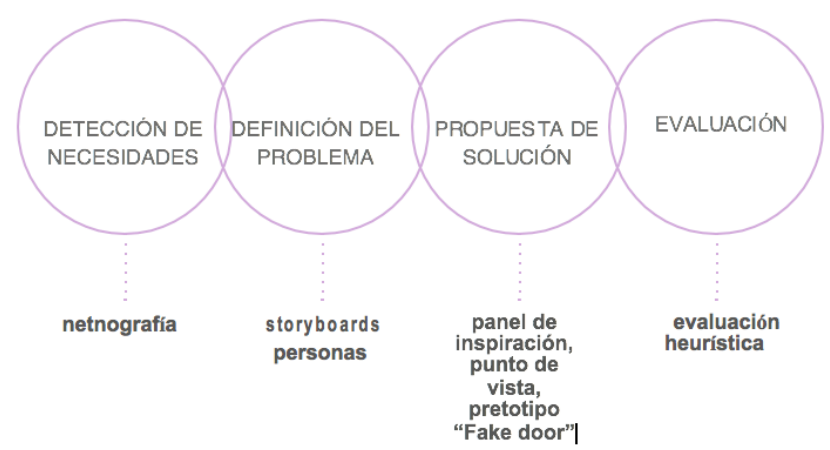

Figura 1. Las 4 etapas principales que conforman la metodología empleada en el desarrollo de BIOLETA.

La metodología propuesta para el desarrollo de BIOLETA consta de 4 etapas principales: 1) detección de necesidades, 2) definición del problema, 3) propuesta de solución y 4) evaluación. La detección de necesidades se realizó a partir de la técnica de investigación conocida como netnografía, posteriormente, se definió el problema, y se utilizaron herramientas del enfoque DCU: storyboards y personas. La tercera etapa, consistió en la propuesta de solución que se desarrolló a partir de un panel de inspiración, que permitió la construcción del punto de vista (eje rector de la solución), seguido de la estrategia del pretotipo y finalmente, la elaboración del prototipo. Por último, para la etapa de evaluación, se probó el prototipo con 5 usuarios siguiendo las 10 heurísticas propuestas por Jakob Nielsen [14].

En las siguientes secciones se detalla cada una de las etapas realizadas.

\subsection{Detección de Necesidades}

La cuarentena causada por la pandemia del COVID-19 cambió muchos aspectos de la vida incluida la manera en la que se realiza investigación con los usuarios. Con el fin de tener un mejor entendimiento de la situación de los artesanos y con la imposibilidad de llevar a cabo observación contextual, se utilizó la netnografía. El término netnografía fue utilizado por primera vez por el experto en marketing Robert Kozinets (1995) ${ }^{9}$, al principio resultó de gran importancia para la creación de insights y mensajes publicitarios, sin embargo, con la situación actual se ha convertido en la única forma llevar una investigación con distancia física. Como sugiere Del Fresno (2011), la netnografía permite acceder a la comprensión y las claves culturales de esferas sociales que están ocultas socialmente, minoritarias o difíciles de localizar físicamente [4]. Esta es una técnica que busca entender las interacciones sociales y los comportamientos humanos en contextos de comunicación digital. Asimismo, durante la investigación se fue consciente de las debilidades y preocupaciones éticas asociadas con el uso de técnicas netnográficas, especialmente cuando parte del problema es la brecha digital que existe en la comunidad que se estaba abordando. Por lo tanto, la realización de la investigación fue una metainvestigación porque (al menos directamente) era complicado estudiar a través de los medios digitales a una comunidad que por varias razones ha sido excluida de la tecnología.

\footnotetext{
${ }^{8} \mathrm{https}: / / \mathrm{maps} . \mathrm{me} /$

${ }^{9}$ https://kozinets.net/about
} 
El desarrollo del proyecto no se detuvo a pesar de la imposibilidad de llevar a cabo técnicas de estudio de campo etnográfico (observación y entrevistas contextuales). Aunque no se pudieron realizar entrevistas con vendedores ambulantes, se realizaron observaciones, a través de las redes sociales. A partir de ellas se encontraron posts con fotos de artesanos que intentaban intercambiar su trabajo por comida. Por ejemplo, la solicitud de cambio de artesanías por comida es explícito en las fotografías de la Figura 2. Era claro que los artesanos tenían problemas para satisfacer sus gastos más básicos, al pertenecer a comunidades cuyos medios de subsistencia dependen de las ventas en la calle. Debido a la situación de COVID-19, tuvieron una reducción significativa en el tráfico peatonal lo que impidió que sus productos se vendieran. ¿Cómo podrían los artesanos continuar vendiendo su trabajo durante esta emergencia y generar ingresos sin tener que (casi) regalar su trabajo?

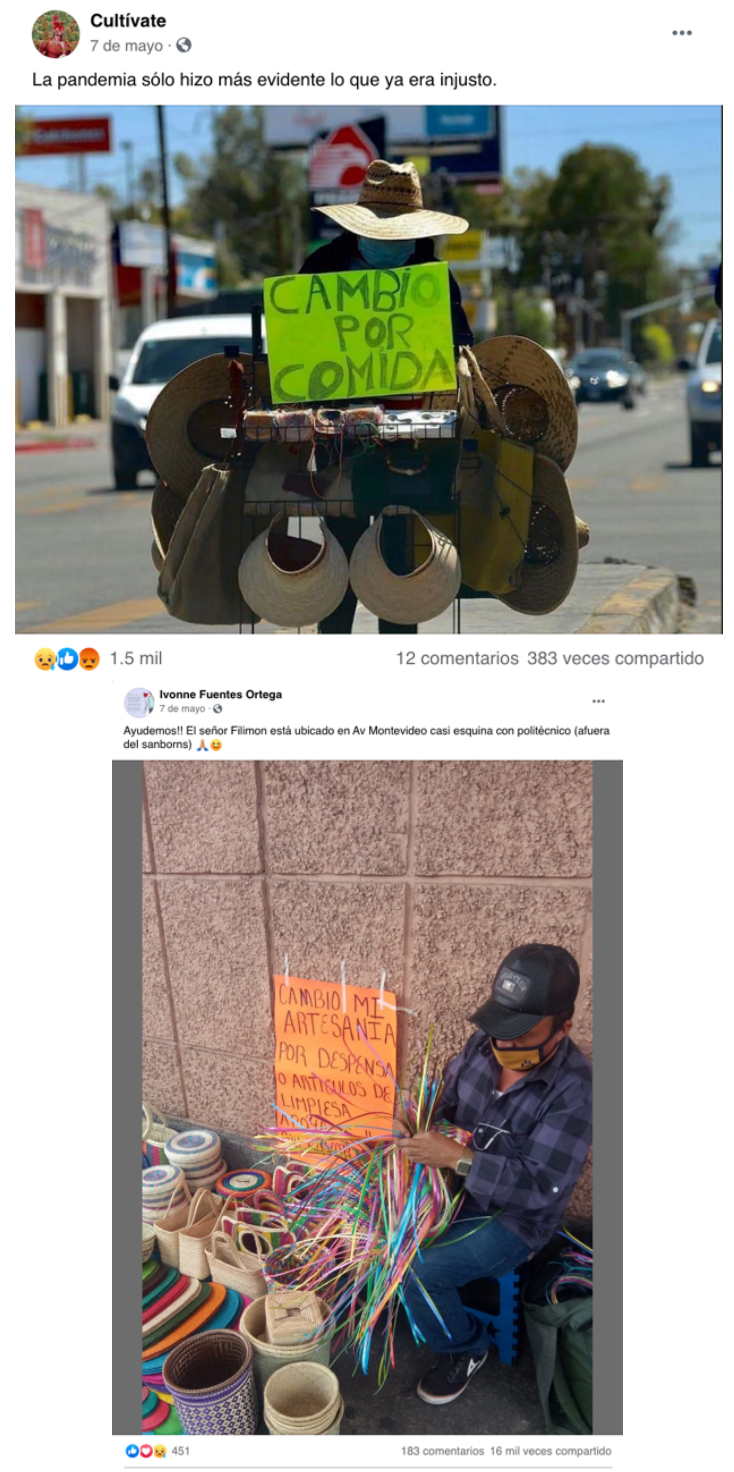

Figura 2. Ejemplos de dos posts solicitando el intercambio de artesanías por comida.

\subsection{Definición del Problema}

En esta etapa del proceso, se delimitaron todas las necesidades que se encontraron para seleccionar solo una que pudiera tener una posible solución en un período corto de tiempo. Para ello, se utilizó la metodología de personas (ver Figuras 3, 4 y 5) y los storyboards (ver Figura 6). Ambas son herramientas de DCU que desde la metodología ágil [2] se consideran como un marco de trabajo donde los objetivos son mejorar la eficiencia en la producción y la calidad de productos finales.

Personas es un método introducido por Alan Cooper [1] para proporcionar una forma precisa de pensar y comunicarse acerca de cómo se comportan los usuarios, cómo piensan, qué desean lograr y por qué. Personas son arquetipos construidos con base en los comportamientos y las motivaciones de personas reales. Para el desarrollo de BIOLETA, se realizó una observación y entrevistas no estructuradas de manera digital a distintas personas para conocer sus comportamientos y motivaciones. Posteriormente, se crearon tres diferentes arquetipos de personificaciones según los tipos de usuarios identificados.

La primera persona corresponde a Josefina, representada en la Figura 3. Josefina es una artesana que vende su trabajo en las calles y que no ha podido vender durante la cuarentena.
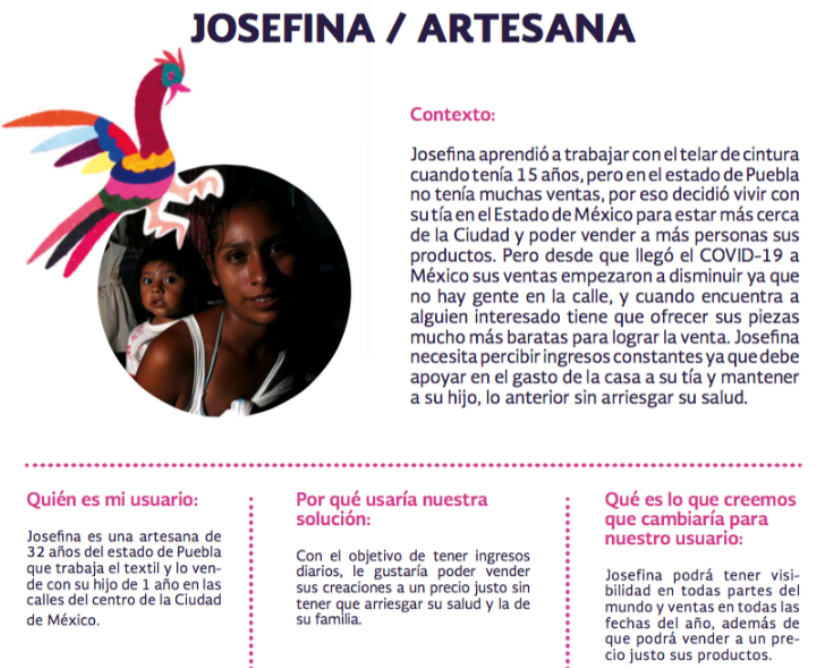

Figura 3. Persona correspondiente al productor artesano.

Lucía es la persona 2, una mujer que ama las artesanías y que le gustaría comprar para poder ayudar a los artesanos, pero no sabe cómo encontrarlas o cómo hacerlo durante la cuarentena (ver Figura 4). 


\section{LUCÍA / COMPRADORA DE ARTESANÍAS}

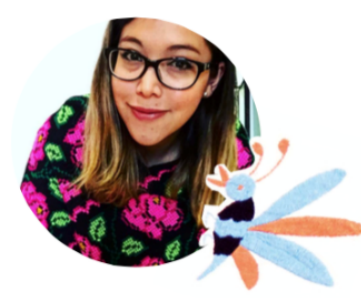

Contexto:

A Lucía le gusta mucho comprar artesanías, siempre que va de viaje a otro estado del país compra alguna artesania; el bordado de Tenango Oaxaca son sus preferidos.

Hace unos meses, su novio le regaló un caballito de palma que le compró a un hombre que vende artesanías cerca de la Alameda, a Lucíale encantó y le gustaría comprar más, pero el día que fue a buscarlo no lo encontró.

Quién es mi usuario:

Lucía tiene 32 años, eseditora delibros de primaria y vive con su pareja y dos perros.

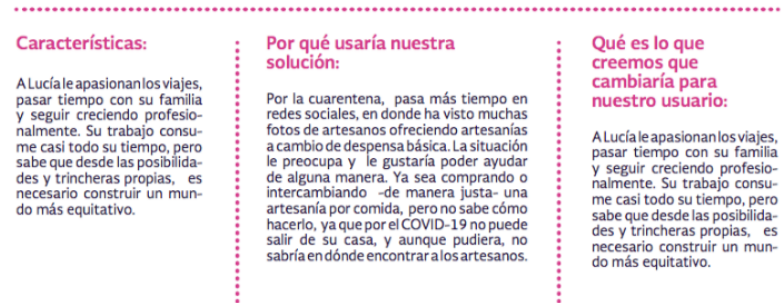

Figura 4. Persona correspondiente a la clienta que le gustan las artesanías, pero que no sabe acerca de ellas.

La persona 3 es Ana Paulina, una mujer a la que le gustan las artesanías, pero no está familiarizada con el valor simbólico y la cantidad de trabajo que requieren (ver Figura 5).

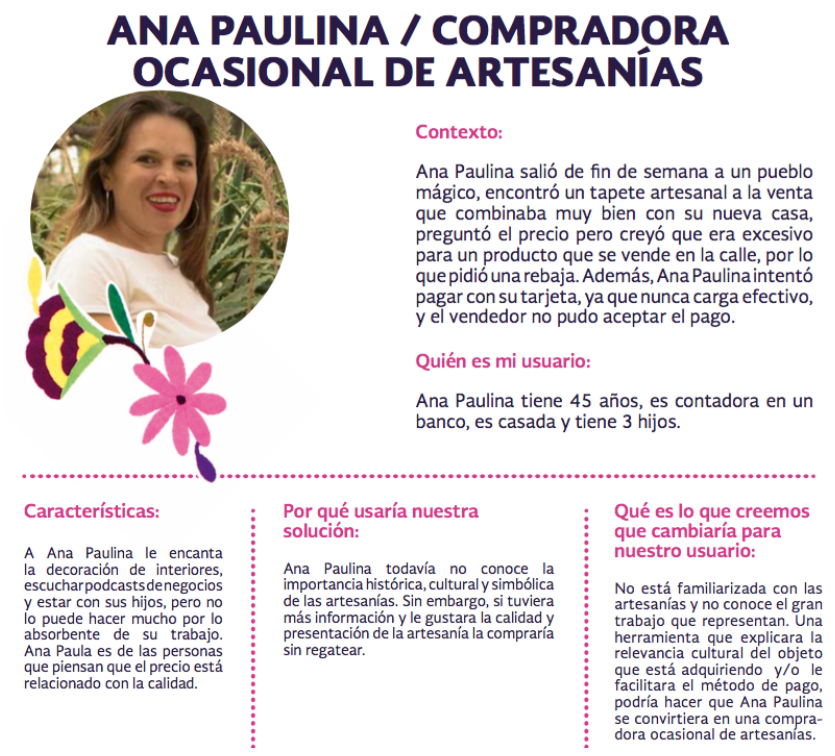

Figura 5. Persona correspondiente a la compradora ocasional de artesanías.

El ejercicio realizado con personas permitió que el equipo se enfocara durante todo el desarrollo del proyecto en quiénes eran los usuarios finales y lo que esperaban de BIOLETA. Esto permitió crear un producto final en el que se propicia una experiencia idónea que podrá aumentar la posibilidad de ventas para artesanos, como empatizar con el usuario.

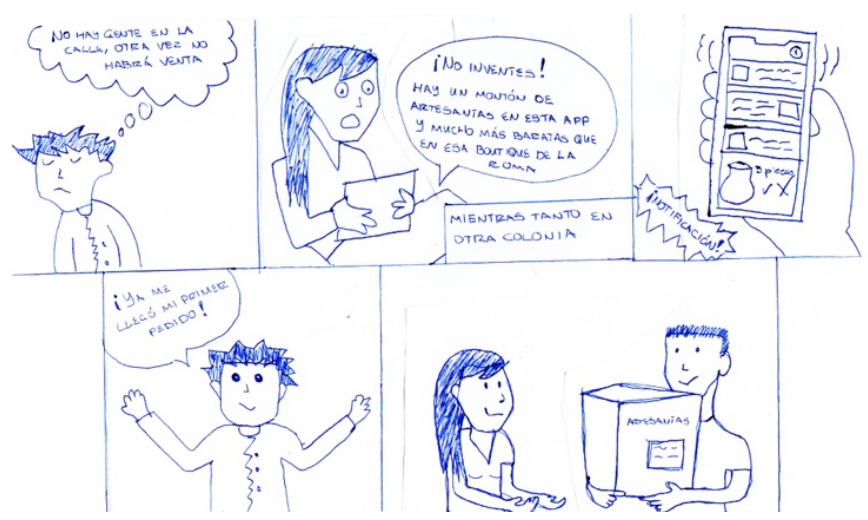

Figura 6. Storyboard mostrando el problema de venta por parte de los artesanos y su posible solución.

Para esta parte del proceso, cada miembro del equipo dibujó un storyboard, una herramienta del DCU que representa gráficamente a un usuario y las motivaciones del usuario para usar una interfaz. Los storyboards reflejan la amplia gama de posibilidades hacia dónde puede ir una posible solución. Es un ejercicio de comunicación que permite que entre los miembros del equipo haya un entendimiento de cómo se ve el problema y hacia dónde puede ir la solución. El storyboard no muestra cómo funciona una aplicación o sistema, se enfoca únicamente en mostrar las emociones del usuario y el resultado satisfactorio del problema. Entre las soluciones propuestas, donde cada miembro del equipo planteó un camino diferente, se encontraron las siguientes: una aplicación de comercio justo, realidad virtual para visualizar la dificultad del trabajo artesanal y una plataforma para intercambiar artesanía por alimentos. Un ejemplo de storyboard se presenta en la Figura 6 en la que el artesano no puede vender y hay una posible compradora que a partir de una aplicación logra vincularse con el artesano y comprarle su artesanía.

A partir de los storyboards surgieron coincidencias y diferencias acerca de las propuestas, entre los miembros del equipo desarrollador, que ayudaron a visualizar e identificar otras maneras de resolver el mismo problema. Para hacer una propuesta de solución era necesario seleccionar lo mejor de cada uno teniendo un enfoque holístico.

\subsection{Propuesta de Solución}

Para poder llevar a cabo un proceso más estructurado y crear un proyecto inclusivo, se utilizaron más herramientas provenientes del DCU. Esto permitió facilitar el proceso y encontrar un punto de vista a partir del uso de un panel de inspiración. Para poder medir la viabilidad de la idea se optó por usar un pretotipo [19].

\subsubsection{Panel de inspiración}

El panel de inspiración tiene como uno de sus propósitos el incentivar la creatividad al analizar el estado del arte. Este consiste en buscar aplicaciones, objetos, todo lo que puede servir de inspiración para una solución. Asimismo, del ejercicio se desprenden palabras clave que pueden caracterizar la solución. A partir de ello, el equipo de trabajo guía el proceso siempre enfocándose en lo que considera que es prioritario a destacar en la solución. En este caso las palabras clave fueron: empatía, colaboración, comercio justo, colores, cultura, comunidades indígenas y reevaluación. Esta actividad también ayudó a la construcción del punto de vista que sirvió como apoyo en el proceso de toma de decisiones y el desarrollo del proyecto: "las personas interesadas en comprar artesanías deberían tener varias opciones 
disponibles sin intermediarios. De esta manera, el artesano puede obtener un pago mejor y más justo además de promover la revalorización del trabajo artesanal". Este punto de vista está formulado por dos ejes: el comercio justo y la revalorización de la artesanía tradicional.

En búsqueda de inspiración, se encontraron algunos sitios web dedicados a la venta de trabajos artesanales, pero la mayoría de ellos son intermediarios entre los artesanos y los clientes (ver sección 2). A partir de las inspiraciones, se llegó a un consenso, crear una plataforma digital que eliminara a los intermediarios e incluyera a todos los artesanos, incluso aquellos que no tienen presencia digital. BIOLETA, llamada así por la biodiversidad que requieren los procesos artesanales, pretende ser una plataforma que albergue a una red de artesanos mexicanos presentes en todo el país. Los artesanos con presencia digital pueden registrar su información de contacto, de redes sociales, una descripción completa de su trabajo y la cantidad de tiempo que requiere cada una de las piezas que realizan. De igual manera, la aplicación tiene un directorio para encontrar artesanos por especialidad; además, el valor de las artesanías está reflejado en la cantidad de horas que necesita cada objeto para su realización.

Para los artesanos que no tienen acceso a dispositivos digitales (como es el caso de muchos vendedores ambulantes) BIOLETA tiene la opción de "mapeo colaborativo", lo que significa que las personas interesadas en productos artesanales tienen la opción de registrar a los artesanos que conocen: su geolocalización, información acerca de su artesanía y datos de contacto. Esta contribución es una solución a corto plazo para incluir a los vendedores ambulantes de productos artesanales en el mundo digital con el propósito inmediato de aumentar las ventas durante la crisis económica que enfrenta el mundo. Sin embargo, el objetivo final es crear un comercio más justo, consciente y respetuoso por la artesanía tradicional y por las personas que la crean.

\subsubsection{Pretotipo}

Para tener una perspectiva diferente y evaluar si la idea de BIOLETA era plausible, se creó un pretotipo. La técnica de pretotipado fue desarrollada por Alberto Savoia como una forma de probar una idea de producto de forma rápida y económica mediante la creación de versiones extremadamente simplificadas de ese producto [19]. Para BIOLETA, se utilizó la técnica de la puerta falsa (fake door) que consiste en validar una idea (puede ser un producto, un servicio o una función) y mostrar a los usuarios una opción, aunque esta no exista en realidad. Se necesitaba una opción viable y técnicamente factible en un periodo muy breve, por lo que se creó una página de Facebook ${ }^{[1]}$ para saber si el desarrollo de la plataforma era relevante y si ayudaría a la necesidad detectada de poder vender productos artesanales sin intermediarios. El tiempo para la evaluación fue muy corto, por lo que se pagó para obtener la cantidad mínima de publicidad diaria que ofrece Facebook. De esta manera, se pudo llegar a un público más diverso y no solo conocer la opinión de los contactos, lo que podría dar comentarios sesgados. Se cargaron dos publicaciones en la página de Facebook: la primera fue para promocionar la página e invitar al público a formar parte de BIOLETA (ver Figura 7) y la segunda era para solicitar completar una encuesta, realizada con los formularios de Google, y así poder obtener más información sobre los usuarios potenciales.

Después de tres días, se analizaron los resultados y se obtuvo información importante, especialmente de que el concepto de artesanía debía ser delimitado ya que muchos de los comentarios que se recibieron fueron de personas que crean productos hechos a mano, pero no son expresiones de una herencia cultural. Fue un ejercicio muy útil, aunque fue emocionalmente difícil porque permitió dar una visión general de la urgente necesidad económica que experimentan las personas. El ejercicio del pretotipo no ofrecía soluciones a corto plazo, lo cual fue una necesidad expresada constantemente durante el tiempo que se publicó una falsa solución.

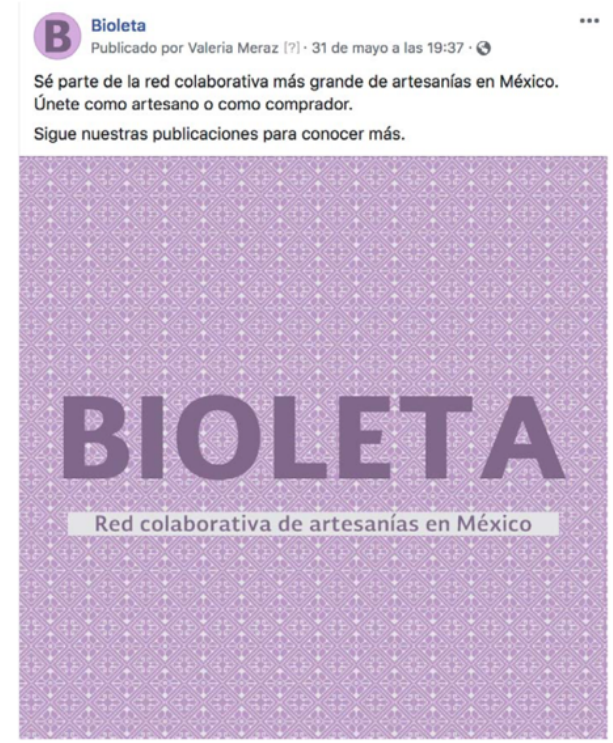

Figura 7. Post en Facebook dentro de la página de BIOLETA.

Las estadísticas de la página de Facebook de BIOLETA mostraron lo siguiente: en 72 horas la página alcanzó 1609 personas, 52 interacciones, 6 comentarios y 3 veces fue compartida la primera publicación. Por correo personal se recibieron 12 mensajes en total. El $53 \%$ de las personas interesadas que interactuaron con la publicación fueron mujeres, con la edad más alta de rangos que oscilan entre 25-34, 45-54 y 55-64 años. En cuanto a los hombres, se obtuvo un $46 \%$ de compromiso y los rangos de edad más altos fueron de 18-24 y 25-34. La encuesta fue respondida por 22 personas donde el $93.5 \%$ declaró que una plataforma para comprar o vender artesanía sería útil. Por su parte, $85.2 \%$ (de los compradores potenciales) respondió que ayudaría saber la geolocalización y la información de contacto de los artesanos sin presencia digital. A su vez, el 54.8\% respondió que regateaban o que les habían regateado al comprar sus productos artesanales.

El tiempo y la muestra fueron insuficientes, pero fue un buen punto de partida que permitió tener una perspectiva más amplia del contexto y las necesidades. Hubiera sido ideal obtener una muestra más grande, especialmente de artesanos, pero el ejercicio respalda que una plataforma sin intermediarios era un proyecto pertinente.

\section{Principales resultados}

El prototipo final de BIOLETA consiste en una aplicación para apoyar las ventas y la revaloración del trabajo artesanal mediante el registro de artesanos mexicanos y la colaboración de personas interesadas en la artesanía para registrar a los artesanos que no tienen presencia digital. Por ello, el prototipo consiste en:

- $\quad$ Pantalla de inicio de sesión: pantalla que permite el acceso para un nuevo usuario y para el ya registrado. El botón de inicio invita a la acción de colaborar, reforzando la idea de una red colaborativa. 
- Menú principal: consta de 6 apartados que comprenden el directorio artesanal, el mapa colaborativo, las técnicas artesanales, el salón virtual, sobre BIOLETA y mi perfil (Figura 8).

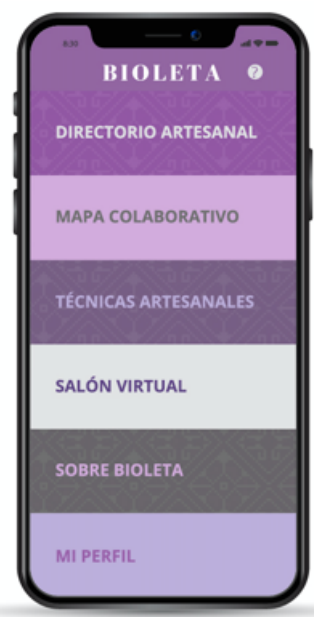

Figura 8. Menú principal.

- Directorio artesanal: esta pantalla muestra un catálogo de diferentes artesanos (Figura 9). El usuario tiene la opción de visualizar cada uno de ellos utilizando la opción "buscar". De esta manera, los usuarios pueden centrarse en trabajos manuales o ubicaciones específicas. La característica más importante de esta pantalla es la cantidad de horas que se tarda en construir una artesanía en lugar del precio expresado con dinero. El propósito de esto es promover la valorización del trabajo de los artesanos. La cantidad de dinero que vale una pieza debe reflejar la cantidad de horas que los artesanos han dedicado a crear lo que los usuarios compran.

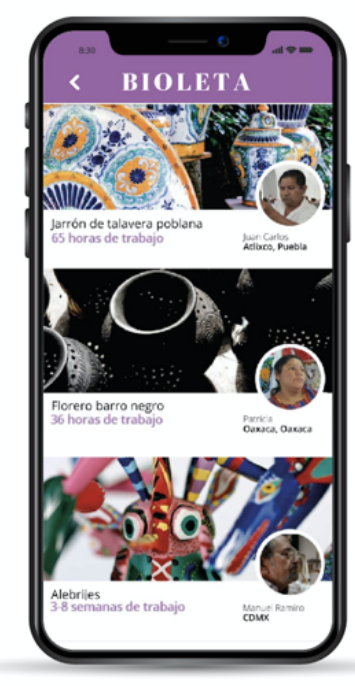

Figura 9. Directorio artesanal.

- Mapa colaborativo: una característica importante de la aplicación es que muestra un mapa y ofrece la opción de "mapear", esto permite al usuario colaborar registrando a un artesano sin presencia digital (ver Figura 10).

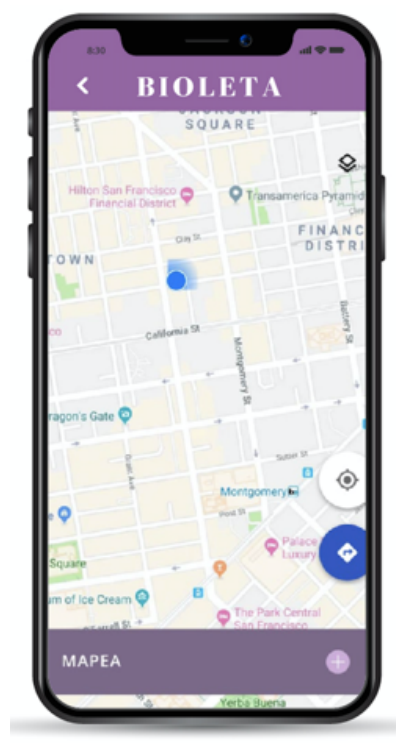

Figura 10. Mapa colaborativo.

- Información acerca de técnicas artesanales: este es un apartado que también se realiza de manera colaborativa y que tiene como objetivo valorizar el trabajo artesanal y dar a conocer su proceso.

- Registro de artesanos: esta parte de la aplicación muestra las opciones que tiene el usuario para registrar a un artesano: la geolocalización (si vende en una calle o vecindario específico), el nombre, el lugar de origen y la información sobre la artesanía que realiza. También ofrece la posibilidad de subir fotos del trabajo artesanal (ver Figura 11).

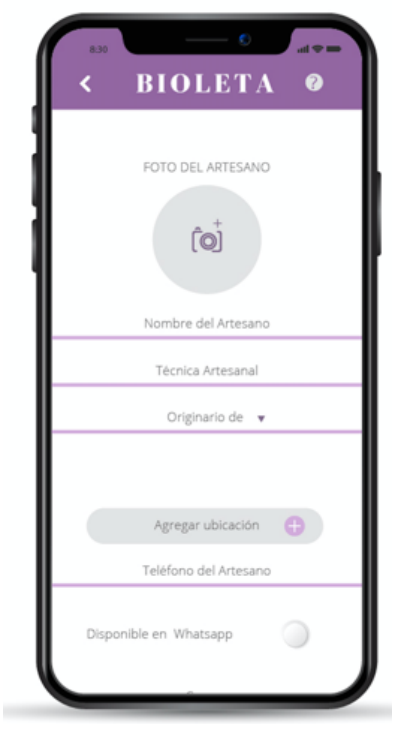

Figura 11. Registro de artesanos.

- Sobre BIOLETA: información acerca del equipo desarrollador. 
- Salón virtual: permite conocer las técnicas y procesos de desarrollo a partir de la disposición de videos en los que aparecen los artesanos (ver Figura 12).

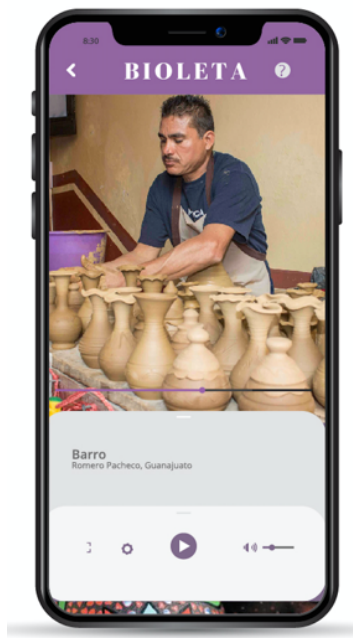

Figura 12. Salón virtual.

- Mi perfil: permite que el usuario pueda cambiar información acerca de su perfil tal como datos personales, fotografía, etc. Igualmente, registra el número de colaboraciones que realiza un usuario de manera a recibir, posteriormente, un reconocimiento (ver Figura 13).

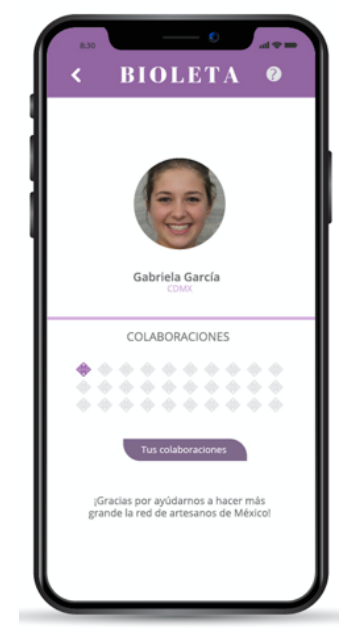

Figura 13. Mi perfil.

\section{Evaluación}

Para comprender cómo iba a interactuar el usuario final con BIOLETA y si podría realizar actividades y tareas con la aplicación, se seleccionaron a cinco usuarios de perfiles distintos [15]: dos posibles compradores no mapeadores, un posible comprador mapeador y un artesano con presencia digital; para evaluar el prototipo con las 10 heurísticas propuestas por Jakob Nielsen [14]:

1. Visibilidad del estado del sistema.

2. Metáforas familiares y lenguaje.

3. Control del usuario y libertad.
4. Consistencia.

5. Prevención de errores.

6. Reconocimiento sobre el recuerdo

7. Flexibilidad y eficiencia de uso.

8. Diseño estético y minimalista.

9. Reconocimiento, diagnóstico y recuperación a partir de errores.

10. Ayuda y documentación.

Las fases de evaluación fueron las siguientes: 1. Entrenamiento: se les proporcionó a los evaluadores la información general del sistema y se les pidió que realizaran las dos tareas principales de la plataforma: colaborar y mapear a un artesano sin presencia digital y encontrar la información de los artesanos de la base de datos; 2. Evaluación: los evaluadores realizaron la tarea y se registraron los resultados; 3. Asignar una calificación: se determinó en una escala ascendente del $0 \mathrm{al} 4 \mathrm{el}$ nivel del problema de usabilidad; 4. Reflexiones finales con el equipo: este ejercicio proporcionó información importante para mejorar BIOLETA, especialmente se encontró que el mapeo no era una opción clara. Al mapear, se pretende que los usuarios colaboren para localizar artesanos en lugar de solo buscarlos. A este respecto, se notó que la palabra "mapeo" no es común entre los usuarios; por lo tanto, además de agregar una pantalla en el prototipo que es más específica y clara con la opción de completar la información sobre la ubicación del artesano, también se incluyó una explicación exhaustiva sobre esta funcionalidad en el menú.

Otras conclusiones se relacionaron con cambios más simples sobre el diseño y la posición de algunos botones para lograr una navegación más intuitiva a través de la aplicación. Por ejemplo, la opción "ayuda" ahora está siempre presente.

\section{Conclusiones y trabajo a futuro}

Este trabajo se centra en proponer una solución para los artesanos que, debido a la brecha digital en México y la pandemia causada por COVID-19, no tienen acceso a una herramienta que les permita vender sus productos a un precio justo. La brecha digital es una consecuencia de las desigualdades sociales y debe abordarse desde una perspectiva holística [13]. Sin embargo, este artículo presenta un primer prototipo y una solución a corto plazo ya que se pretende incluir a más personas en los beneficios de la economía digital.

El DCU marcó la diferencia en el proceso al mejorar el acercamiento entre los usuarios y el equipo de desarrollo. Algunas de las actividades se realizaron a distancia haciendo uso de la netnografía. A su vez, el DCU permitió generar empatía con las personas que participaron. Al diseñar proyectos cuyo objetivo es resolver problemas de la vida real es central incluir a las comunidades para poder generar un impacto social positivo. El ejercicio del pretotipado fue revelador al poder evidenciar la gran necesidad que existe por parte de los artesanos, especialmente durante la pandemia de COVID-19.

A lo largo del proceso de creación de BIOLETA, se descartaron muchas ideas durante la primera fase. La toma de decisiones consideró el tiempo de producción requerido, su complejidad técnica, los desafíos económicos y las dificultades de implementación del prototipo.

Para una segunda etapa de BIOLETA, el objetivo será promover la revalorización del trabajo artesanal. Esto podrá lograrse a partir de material audiovisual que estimule los sentidos del usuario, de modo que su observación llegue a ser una experiencia inmersiva en la elaboración de artesanías. Este ejercicio buscará informar a la audiencia sobre el arduo trabajo requerido para producir una pieza artesanal en términos de valor 
cultural y económico. De esta forma, se mostrará todo el proceso detrás de los productos artesanales: desde la búsqueda de materiales para crear estos productos hasta la venta de estos. Asimismo, se espera abordar el problema del regateo y crear conciencia para eliminarlo. El regateo no es una práctica cultural, sino un reflejo del poco valor que se da al trabajo artesanal.

Por lo anterior, la propuesta de BIOLETA es crear una campaña permanente de difusión al valor del trabajo artesanal y, en consecuencia, la eliminación del regateo. Con este fin, BIOLETA podría convertirse en la plataforma con la red más grande de artesanos en México, a través de un modelo exitoso que ofrezca la posibilidad de replicarse y expandirse a otros países con su propio patrimonio artesanal. Por último, el mapeo colaborativo, idealmente, permitirá crear un sentido de comunidad y aumentar las ventas para los artesanos. Como sociedad, debemos trabajar duro para garantizar que los desarrollos tecnológicos beneficien a todos y reduzcan las brechas de desigualdad.

\section{Referencias}

[1] Cooper, A., Reinmann, R., Cronin, D., and Noessel, C. About Face: The essentials of interaction design, Wiley Publishing, Inc., Indianapolis. (2007) 720 p.

[2] Duarte, A. O., and Rojas, M. Las metodologías de desarrollo ágil como una oportunidad para la ingeniería del software educativo. Revista Avances en Sistemas e Informática, 5 (2). (2008).

[3] El Universal. Mexico has a sharp digital divide. El Universal, Mexico. (2018).

https://www.eluniversal.com.mx/english/mexico-has-sharpdigital-divide

[4] Del Fresno, M. (2011). Netnografia Investigación, análisis e intervención social online. http://mastor.cl/blog/wpcontent/uploads/2019/07/Del-Fresno-Netnografia.pdf

[5] Fondo Nacional para el Fomento de las Artesanías. (2015). Manual de diferenciación entre artesanía y manualidad. https://www.gob.mx/cms/uploads/attachment/file/107963/Ma nual_diferenciacion_artesania_manualidad_2015.pdf

[6] Fuentes, G. (2019). En 7 años, 23 marcas plagiaron el diseño autóctono de México, y no hay una sola denuncia: activistas. Sin embargo, Mexico. https://www.sinembargo.mx/22-06-2019/3599883

[7] Heraldo de México. (2017) México, tercer lugar a nivel mundial en elaborar artesanias. México. http://bit.ly/2AKQmt6

[8] INEGI. Estadísticas a propósito del día del trabajo datos nacionales (2020).

https://www.inegi.org.mx/contenidos/saladeprensa/aproposit o/2020/trabajoNal.pdf

[9] INEGI. Secretaría de Comunicaciones y Transportes, \& Instituto Federal de Telecomunicaciones. Boletín de Prensa. Encuesta Nacional sobre Disponibilidad y Uso de
Tecnologías de la Información en los Hogares (2017). https://www.inegi.org.mx/contenidos/saladeprensa/boletines/ 2018/OtrTemEcon/ENDUTIH2018_02.pdf

[10] Interaction Design Foundation. User Centered Design. https://www.interaction-design.org/literature/topics/usercentered-design

[11] Kaizer, F. (2020). COVID-19 pandemic exposes global 'frailties and inequalities': UN. UN News. https://news.un.org/en/story/2020/05/1063022

[12] Lutz, E. The many meaning of technology. Cultural Survival. (2005).

https://www.culturalsurvival.org/publications/culturalsurvival-quarterly/many-meanings-technology

[13] Mecinas, M. The digital divide in Mexico: a mirror of poverty. Mexican Law Review. p. 93-102. ISSN 2448-5306. (2016). https://revistas.juridicas.unam.mx/index.php/mexican-lawreview/article/view/10432

[14] Nielsen, J. 10 Usability Heuristics for User Interface Design. (1994).

https://www.nngroup.com/articles/ten-usability-heuristics/

[15] Nielsen, J. Why You Only Need to Test with 5 Users. Nielsen Norman Group. (2000).

https://www.nngroup.com/articles/why-you-only-need-totest-with-5-users/

[16] RENATRA- National Register of Textile Artisans of the Argentine Republic. (2016) UNESCO, Argentina. https://en.unesco.org/creativity/policy-monitoringplatform/renatra-national-register

[17] Rodríguez, D. Una pieza que vale doce horas: la campaña para evitar el regateo al trabajo de los artesanos mexicanos. El País, Mexico. (2018).

https://verne.elpais.com/verne/2018/07/17/mexico/15318590 18_318594.html

[18] Sales Heredia, F. Las artesanías en México. Situación actual y retos. Centro de Estudios Sociales y de Opinión Pública, México. (2013).

http://biblioteca.diputados.gob.mx/janium/bv/cesop/lxii/art_ mex_sitact_re.pdf

[19] Savoia A. Pretotype It: make sure you are building the right it before you build it right. 69p. (2011)

[20] UNESCO. Técnicas artesanales tradicionales. UNESCO. https://ich.unesco.org/es/ tecnicas-artesanales-tradicionales00057

[21] UNITED NATIONS. Coronavirus reveals need to bridge the digital divide. (2020).

https://unctad.org/en/pages/newsdetails.aspx?OriginalVersio $\mathrm{nID}=2322$

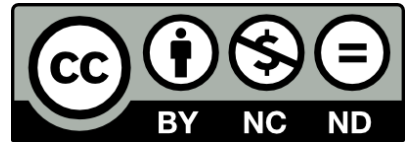

(C) 2020 by the authors. This work is licensed under the Creative Commons AttributionNonCommercial-NoDerivatives 4.0 International License. To view a copy of this license, visit http://creativecommons.org/licenses/by-nc-nd/4.0/ or send a letter to Creative Commons, PO Box 1866, Mountain View, CA 94042, USA. 